\title{
Design and Demonstration of A Compact Full Adder Using Micro-beam Resonators
}

\author{
Sally Ahmed*, Saad Ilyas ${ }^{\dagger}$, Nizar Jaber ${ }^{\dagger}$, Xuecui Zou ${ }^{\ddagger}$, Ren $\mathrm{Li}^{*}$, Mohammad Ibrahim Younis ${ }^{\dagger}$ and Hossein Fariborzi ${ }^{*}$ \\ Email: \{sally.ahmed, saad.ilyas, nizar.jaber, xuecui.zou, ren.li, mohammad.younis, hossein.fariborzi\}@kaust.edu.sa \\ ${ }^{*}$ CEMSE Division, King Abdullah University of Science and Technology, Thuwal, Saudi Arabia. \\ †PSE Division, King Abdullah University of Science and Technology, Thuwal, Saudi Arabia. \\ \#School of Microelectronics and Solid States Electronics, University of Electronic Science and Technology of China (UESTC), \\ Chengdu, China.
}

\begin{abstract}
In this work, we present the design, analytical and finite element simulations and experimental results of a full adder block using microelectromechanical resonators with multiple split electrodes. The device operation is based on modulating resonance characteristics by the digital DC inputs. The proposed full adder is implemented with only two devices, considerably less complex than standard CMOS designs which require 24 or more transistors. While the current device has a 0.1 $\mathrm{kHz}$ speed and energy/operation in $\mathrm{pJ}$, we show that by careful optimization and scaling of the devices, the speed can be increased to $\mathrm{MHz}$ and the energy can be reduced to sub-fJ, which shows the potential of this technology for ultra-low power applications with moderate processing requirements. The device is fabricated using silicon-on-insulator wafer with conventional surface micromachining techniques. The implemented circuit is CMOS compatible which allows its monolithic integration with other CMOS-based circuits. The micro-beam resonator operates at room temperature and moderate pressure of $700 \mathrm{mTorr}$.
\end{abstract}

Keywords-Mechanical Computation; Doubly-clamped Microbeam Resonator; Full Adder; Electro-static Softening Effect

\section{INTRODUCTION}

The continuous miniaturization in complementary-metaloxide-semiconductor (CMOS) technology has led to improvements in the performance and energy efficiency of integrated circuits in the past few decades. However, further improvements by scaling are hindered by the well-known issues of leakage and increased power density. Therefore, alternative computing schemes are sought after. Micro/Nanoelectromechanical systems (M/NEMS) have revived the notion of mechanical computing, offering competitive advantages over CMOS transistors, such as zero off-state leakage and low power consumption. Mechanical computing devices can be classified into two categories: contact and noncontact based devices. M/NEMS Relays represent the contact based category and they have been used to implement basic logic gates [1-2] and more complex arithmetic blocks like adders [3] and multipliers [4]. Although they have virtually zero leakage current and some variations of them are cascadable, they suffer from contact reliability issues, contact oxidation and high contact resistance when scaled [5-6]. On the other hand, micro-resonators, which represent the noncontact based devices, do not suffer from these issues as their operation is based on the amplitude or the phase of the vibrating structure. The speed of the resonators can reach $\mathrm{MHz}$ or $\mathrm{GHz}$ regime with careful device engineering and scaling. Furthermore, this technology allows runtime reprogrammability and reduces system complexity and energy consumption. Micro-resonator based logic gates and memory devices have recently been demonstrated. An XOR gate has been implemented using piezoelectric effect [7]. Reprogrammable logic gates have been implemented using mixed-frequency excitation [8], electro-thermal [9-10] and electrostatic [11-12] frequency modulation. In addition, a universal reversible logic gate (a Fredkin gate) has also been demonstrated using electrostatically coupled resonators [12].

However, complex arithmetic blocks have not been demonstrated using micro-resonator technology. In this work, we demonstrate an essential arithmetic block, the full adder, which is used in performing addition, subtraction, multiplication, address calculations and many other functions. A single bit full adder is demonstrated using two microresonators with multiple split electrodes, which is significantly less than the required transistors of any standard CMOS full adder cell [13].

\section{The Full AdDER DesigN}

\section{A. Device Structure and Operation}

The device consists of a laterally actuated doubly-clamped micro-beam with three fixed identical partial electrodes on each side of the beam as shown in Fig. 1. The electrodes near the beam anchors (corner electrodes) are used for the digital inputs while the two middle electrodes are used for electrostatically actuating the beam and capacitively detecting its output. The operation of the proposed device is based on a shift in the resonance frequency, induced by application of DC inputs, and the electrostatic softening effect. The corner electrodes were chosen for the digital inputs to maintain the symmetry of the device and balance the DC loading effect.

The expected shift in the resonance frequency due to the digital DC inputs can be predicted by solving the eigenvalue problem for the micro-beam using a lumped parameter model [14]. The un-damped equation of motion of the system shown in Fig. 1(b) is given by (1) where $m$ is the mass, $k$ is the stiffness, $A$ is the overlap area between one electrode and the beam, and $\varepsilon$ is the relative permittivity constant. $V_{D C}$ is the 


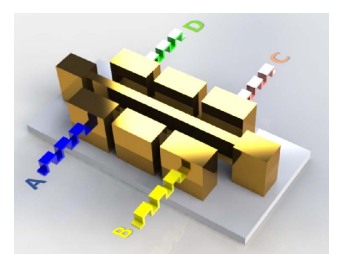

(a)

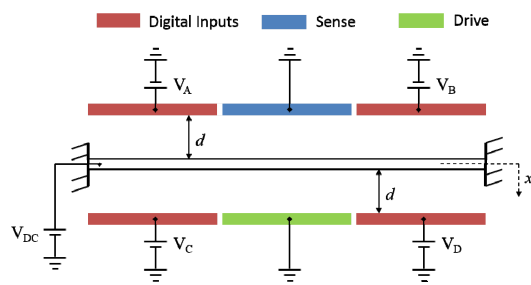

(b)

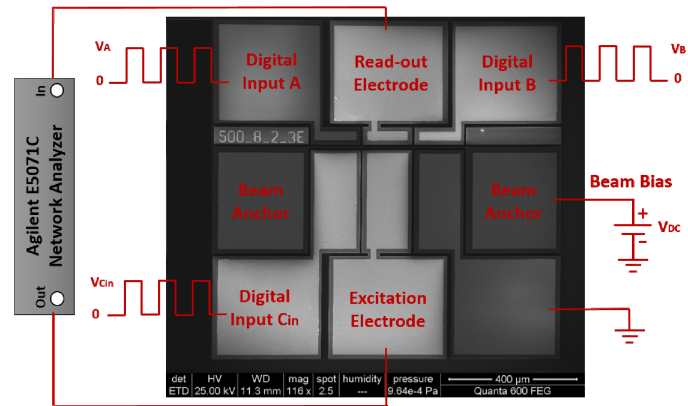

(c)

Fig. 1: (a) 3D Schematic of the micro-resonator, (b) 2D Schematic (c) Scanning electron microscopy (SEM) image of the device with the experimental setup. The beam is $500 \mu \mathrm{m}$ long, $2.3 \mu \mathrm{m}$ wide, $30 \mu \mathrm{m}$ thick and the air-gaps are $8 \mu \mathrm{m}$.

beam bias, $V_{\text {Drive }}$ and $V_{\text {Sense }}$ are the effective DC voltages on the drive and sense electrodes which are much smaller than $V_{D C}$ and can be approximated to zero. $V_{A}, V_{B}, V_{C}$, and $V_{D}$ are the digital inputs.

$m \ddot{x}+k x=\frac{\varepsilon A}{2}\left[\begin{array}{l}\frac{\left(V_{D C}-V_{D r i v e}\right)^{2}}{(d-x)^{2}}-\frac{\left(V_{D C}-V_{\text {Sense }}\right)^{2}}{(d+x)^{2}}-\frac{\left(V_{D C}-V_{A}\right)^{2}}{(d+x)^{2}} \\ -\frac{\left(V_{D C}-V_{B}\right)^{2}}{(d+x)^{2}}+\frac{\left(V_{D C}-V_{C}\right)^{2}}{(d-x)^{2}}+\frac{\left(V_{D C}-V_{D}\right)^{2}}{(d-x)^{2}}\end{array}\right]$

The resonance frequency of the resonator can be expressed as [11]

$\omega=\sqrt{\frac{k}{m}-\frac{\varepsilon A}{m}\left[\begin{array}{l}\frac{V_{D C}{ }^{2}}{(d-\delta)^{3}}+\frac{V_{D C}{ }^{2}}{(d+\delta)^{3}}+\frac{\left(V_{D C}-V_{A}\right)^{2}}{(d+\delta)^{3}}+\frac{\left(V_{D C}-V_{B}\right)^{2}}{(d+\delta)^{3}} \\ +\frac{\left(V_{D C}-V_{C}\right)^{2}}{(d-\delta)^{3}}+\frac{\left(V_{D C}-V_{D}\right)^{2}}{(d-\delta)^{3}}\end{array}\right]}$

where $\delta$ is the static solution of (1) for the given DC bias conditions.

It is observed from (2) that five different resonance frequencies corresponding to five different cases (shown in Table 1) are obtained. Theoretically, the digital combinations containing the same number of ones result in close resonance frequencies for the beam.

We further perform a finite element simulation (FEM) using COMSOL software [15] to verify the results. Our aim is to build a full adder which requires only 3 input electrodes for complete operation. Therefore only three corner electrodes are used as inputs while the fourth one is grounded. A parametric sweep is performed to obtain the resonance frequencies of the beam for the eight different combinations from 000 to 111 . The digital input (1) and (0) are defined as $25 \mathrm{~V}$ and $0 \mathrm{~V}$, respectively. The output digital (1) is defined as the on-
TABLE I. RESONANCE FREQUENCIES CORRESPONDING TO DIFFERENT INPUT COMBINATIONS.

\begin{tabular}{|c|l|l|}
\hline Case & \multicolumn{1}{|c|}{$\begin{array}{c}\text { Resonance } \\
\text { Frequency }\end{array}$} & \multicolumn{1}{|c|}{ Input Combinations (ABCD) } \\
\hline All-Zeros & $f_{0}$ & 0000 \\
\hline Single-one & $f_{1}$ & $0001-0010-0100-1000$ \\
\hline Two-ones & $f_{2}$ & $0011-0101-1001-0110-1010-$ \\
& & 1100 \\
\hline Three-Ones & $f_{3}$ & $1110-1101-1011-1110$ \\
\hline Four-Ones & $f_{4}$ & 1111 \\
\hline
\end{tabular}

resonance signal while output digital $(0)$ is the off-resonance signal. The beam bias is $60 \mathrm{~V}$. Fig. 2 shows that analytical model, COMSOL simulation and experimental results have the same trend in frequency shift between the different cases. However, slight deviations are observed due to the approximations of theoretical models employed and not accounting for residual stresses in the beam. Although analytical model and COMSOL simulations show that all single-one combinations $(100,010,001)$ result in almost the same resonance frequency, the experimental data shows that the resonance frequency resulting from the 001 case is slightly higher than the other two combinations. However, this frequency shift is very small and the frequency of operation is chosen to be at the intersection of resonance responses of the three single-one cases. On the other hand, in the two-ones combinations, we notice that the resonance frequency when two high inputs lie on the same side of the beam (110) is slightly different from the cases where the two ones lie on opposite sides of the beam (011 and 101). This is due to the fact that each loading condition results in a different total DC loading on the beam (2). However, the device loading conditions are selected such that the frequency shift between these cases is enough for a full adder operation. Again, the intersection of the frequency responses of these combinations was chosen as the operating frequency of two-ones case.

\section{B. Case Study: Full Adder}

A full adder block has three inputs: the two digits to be added ( $\mathrm{A}$ and $\mathrm{B})$ and a carry-in input $\left(\mathrm{C}_{\mathrm{in}}\right)$. It has two outputs, the sum $(\mathrm{S})$ and the carry-out $\left(\mathrm{C}_{\text {out }}\right)$. The sum is a 3-input XOR gate while the carry can be described by equation (3)[16]

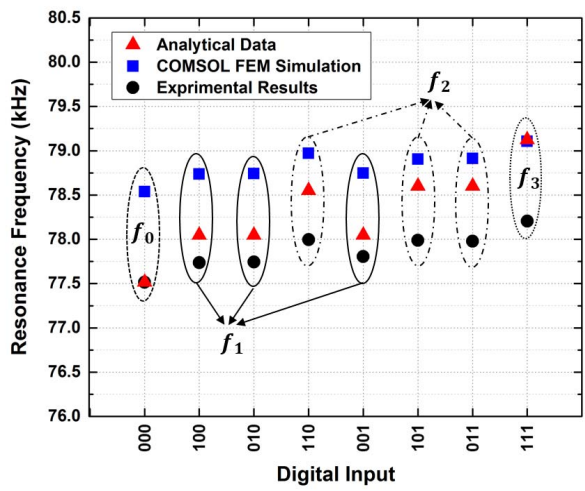

Fig. 2: Analytical and COMSOL simulations and experimental data showing the resonance frequencies of the beam for different digital input combinations. 


$$
C_{\text {out }}=A B+A C_{\text {in }}+B C_{\text {in }}
$$

In part (A), we showed the input vectors containing the same number of ones result in very close resonance frequencies. Each output of the full adder requires one microresonator device. While two devices are identical, the applied frequencies determine which output is obtained. To obtain the sum, two signals with frequencies $f_{l}$ and $f_{3}$ are applied simultaneously such that if the input vector contains an odd number of ones, the beam resonates and a high $S_{21}$ signal is obtained. Similarly, to obtain the carry output, two ac signals with frequencies $f_{2}$ and $f_{3}$ are applied simultaneously.

\section{EXPERIMENTAL RESULTS}

Fig. 1(c) shows a scanning electrode microscopy (SEM) image of the fabricated micro-resonator device and the experimental setup. As mentioned earlier, two devices are required for the full adder operation. As we mentioned earlier each device can be reprogrammed, so for simplicity, only one device was used to obtain both results. A time-varying signal is applied to the drive electrode from the network analyzer to actuate the beam. The output is detected at the sense electrode and fed back to the network analyzer, after passing through a low noise amplifier (LNA), for $S_{21}$ parameter measurement. The digital inputs were generated by Arduino micro-controller followed by a simple level shift circuit to shift the digital (1) value from $5 \mathrm{~V}$ to $25 \mathrm{~V}$.

Fig. 3 shows the frequency response of the beam for the eight different input combinations where $f_{0}=77.5 \mathrm{kHz}$, $f_{l}=77.75 \mathrm{kHz}, f_{2}=78 \mathrm{kHz}$, and $f_{3}=78.2 \mathrm{kHz}$. After identifying the operating frequencies for the different cases, a $5 \mathrm{mV}$ (rms) ac signal with a fixed operating frequency is applied to the drive electrode. Although obtaining the sum or the carry requires the simultaneous application of two signals with two different frequencies, a single frequency was applied at a time due to limitations in the experimental setup and the fact that we only have one drive electrode in this generation of microresonators. The network analyzer was used to find the time response of the $S_{21}$ parameter at the applied frequency.

Fig. 4 shows the time response of the circuit. The sum output is obtained in two consecutive steps, first by applying the frequency of single one $\left(f_{l}\right)$ to the drive electrode followed by applying the frequency corresponding to the all-ones case $\left(f_{3}\right)$. The sum $S_{21}$ output is high only if a single one (blue) or three ones (green) exist in the input vector. Similarly, the carry-out is obtained by applying $\left(f_{2}\right)$ followed by $\left(f_{3}\right)$. Similarly, the carryout $S_{21}$ output is high only if two-ones (orange) or three ones (green) exist in the input vector.

\section{DISCUSSION}

It is important to highlight the advantages and address some of the challenges of using micro-electromechanical resonators in digital circuit design. In this section, the speed, energy per operation, and area consumption are discussed. Other properties such as frequency stability, temperature stability and alternative sensing techniques have been discussed in a previous work [13].

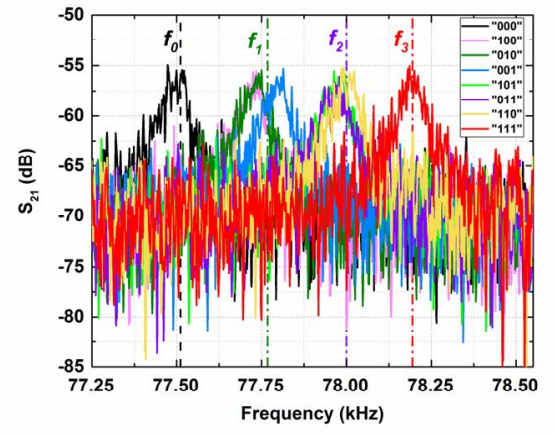

Fig. 3: Frequency response of the micro-resonator for different digital input combinations.

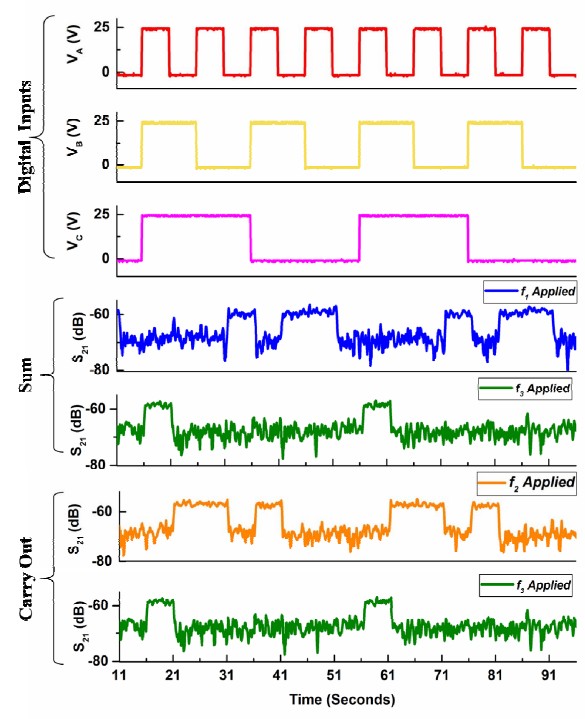

Fig. 4: Time responses of the Sum and the Carry obtained by applying the operation frequencies consecutively to the drive electrode.

The micro-resonator speed is determined by $f / Q$ [17], where $Q$ is the quality factor of the resonator, approximately 1000 when operating at $700 \mathrm{mTorr}$, while $f$ is the frequency of operation. Therefore, the speed is limited to $\approx 0.1 \mathrm{kHz}$. The speed can be significantly improved by designing the resonator to have resonance frequency in the $\mathrm{GHz}$ range, and a lower quality factor that is just enough to differentiate between logic (1) and logic (0) output signals as shown in Table II.

Another important evaluation metric is the consumed energy per logic operation which consists of two components: switching energy $\left(E_{\text {Switching }}\right)$ and ac activation energy $\left(E_{\text {Activation }}\right)$

$$
\begin{aligned}
& E_{\text {Total }}=E_{\text {Switching }}+E_{\text {Activiation }} \\
& E_{\text {Switching }}=\frac{1}{2} C\left[\begin{array}{l}
\left(V_{D C}{ }^{2}-\left(V_{D C}{ }^{2}-V_{A}{ }^{2}\right)\right)+ \\
\left(V_{D C}{ }^{2}-\left(V_{D C}{ }^{2}-V_{B}{ }^{2}\right)\right)+ \\
\left(V_{D C}{ }^{2}-\left(V_{D C}{ }^{2}-V_{C_{i n}}{ }^{2}\right)\right)
\end{array}\right]
\end{aligned}
$$

where $C$ is the capacitance between one partial electrode and the beam $(\approx 5.17 \mathrm{fF})$. The switching energy per operation can 
be calculated using (5), which has three terms, each term represents the energy stored in the capacitor between the input electrode and the beam. The switching energy per operation for one micro-resonator was calculated for the eight different combinations and the average is $14.08 \mathrm{pJ}$. Therefore, a single bit full adder consumes $28.16 \mathrm{pJ} /$ operation as it consists of two micro-resonators. The switching energy can be significantly reduced by decreasing the capacitance and the operating voltages, which is achievable by careful shrinking and optimization of the resonator dimensions.

The ac activation energy can be calculated using (6)

$$
E_{\text {Activation }}=\frac{V^{2}}{Z} \times t_{s}
$$

where $V$ is the rms value of the applied ac signal and $Z$ is the impedance of the micro-resonator [11]. The impedance of the micro-resonator was experimentally found to be around 500 $\mathrm{k} \Omega$. With $\mathrm{V}=5 \mathrm{mV}$ (rms), the activation energy per device is $0.6 \mathrm{pJ}$. The total energy per addition is $29.36 \mathrm{pJ}$. Note that the total energy is dominated by the switching energy. The activation energy can be reduced by reducing the switching time and increasing the impedance of the resonator.

The digital one voltage and the beam bias are chosen such that the frequency shift $(\Delta f)$ between two adjacent states (for example between $f_{0}$ and $f_{l}$ ) is greater than the full width at half maximum power, which is equal to $2 f / Q$ [12]. By using $25 \mathrm{~V}$ for digital (1) and $60 \mathrm{~V}$ for the beam bias, the frequency separation is $\Delta f \approx 200 \mathrm{~Hz}$, which is greater than $\Delta f_{\text {min }}(\approx 158$ $\mathrm{Hz}$ ). If we down scale the device as shown in Table II, the resonance frequency will increase to $0.9 \mathrm{GHz}$. Assuming a $Q$ of 1200, and the required digital one and the beam bias voltages will drop to $5 \mathrm{~V}$ and $15 \mathrm{~V}$, respectively. This reduces the energy/operation by 3 orders of magnitude $(\approx \mathrm{fJ})$ and increases speed to $0.7 \mathrm{MHz}$. In general, increasing the resonance frequency and operating at low quality factor will result in higher operation speed.

Note that building a single bit adder, using an optimized resonator shown in Table II, will reduce the area $91 \%$ compared to standard CMOS mirror adder in $65 \mathrm{~nm}$ Technology.

\section{CONCLUSION}

We have demonstrated the design and operation of a full adder block using micro-resonators. The digital inputs tune the resonance frequency of the beam. The applied frequencies determine the operation of the device. The performance and energy consumption can be significantly improved by careful optimization of the device dimensions and operating conditions. Micro-resonator technology also reduces the complexity of digital circuit design and paves the road for implementing ultra-low power computing devices.

\section{ACKNOWLEDGMENT}

This work was supported by the King Abdullah University of Science and Technology (KAUST) office of sponsored research OSR under Award No. OSR-2016-CRG5-3001.
TABLE II. COMPARISON OF SINGLE BIT ADDER BETWEEN THE TESTED RESONATOR, AN OPTIMIZED SIMULATED RESONATOR AND CMOS MIRROR ADDER IN 65NM TECHNOLOGY

\begin{tabular}{|c|l|l|l|}
\hline & $\begin{array}{c}\text { Tested Resonator } \\
(\mathbf{L}=\mathbf{5 0 0}, \mathbf{w}=\mathbf{2 . 3}, \\
\mathbf{g}=\mathbf{8}, \mathbf{t}=\mathbf{3 0})^{\mathbf{a}}\end{array}$ & $\begin{array}{c}\text { Optimized Resonator } \\
(\mathbf{L}=\mathbf{0 . 6}, \mathbf{w}=\mathbf{0 . 0 5}, \\
\mathbf{g}=\mathbf{0 . 0 5}, \mathbf{t}=\mathbf{0 . 0 5})\end{array}$ & $\begin{array}{c}\text { Mirror } \\
\text { Adder } \text { in } \\
\mathbf{6 5 n m} \text { Tech. }\end{array}$ \\
\hline Area & $20300 \mu \mathrm{m}^{2}$ & $0.7 \mu \mathrm{m}^{2}$ & $32 \mu \mathrm{m}^{2}$ \\
\hline Power & $4.079 \mathrm{nW}$ & $1.4 \mathrm{nW}$ & $2.38 \mu \mathrm{W}$ \\
\hline Speed & $0.1 \mathrm{kHz} @ \mathrm{Q}=1000$ & $0.7 \mathrm{MHz} @ \mathrm{Q}=1200$ & $500 \mathrm{MHz}$ \\
\hline $\begin{array}{c}\text { No. of } \\
\text { Devices }\end{array}$ & 2 resonators & 2 resonators & 28 transistor \\
\hline
\end{tabular}

a. Beam dimensions in $\mu \mathrm{m}$ : $\mathrm{L}$ is the length, $\mathrm{w}$ is the width, $\mathrm{g}$ is the air gap and $\mathrm{t}$ is the thickness.

\section{REFERENCES}

[1] C.-Y. Tsai and T.-L. Chen, "Design, fabrication and calibration of a novel MEMS logic gate," Journal of Micromechanics and Microengineering, vol. 20, no. 9, p. 095021, 2010..

[2] S. Ilyas, A. Arevalo, E. Bayes, I. G. Foulds, and M. I. Younis, "Torsion based universal MEMS logic device."Sens. Actuat. A: Phy, vol. 236, pp. 150-158, 2015.

[3] M. Spencer, F. Chen, C. Wang, R. Nathanael, H. Fariborzi, A. Gupta, H. Kam, V. Pott, J. Jeon, T.-J.K. Liu, D. Markovi'c, E. Alon, and V. Stojanovi'c, "Demonstration of integrated micro-electro-mechanical switch circuits for VLSI applications," IEEE J. Solid-State Circuits, vol. 46, pp. 308-320, 2011.

[4] H. Fariborzi, F. Chen, R. Nathanael, J. Jeon, T.-J.K. Liu, and V. Stojanovic, "Design and demonstration of micro-electro-mechanical relay multipliers," IEEE Asian Solid-State Circuits Conference 2011, 2011.

[5] H. F. Dadgour, M. M. Hussain, A. Cassell, N. Singh, and K. Banerjee, "Impact of scaling on the performance and reliability degradation of metal-contacts in NEMS devices," 2011 International Reliability Physics Symposium, 2011.

[6] H. Fariborzi, F. Chen, R. Nathanael, I.-R. Chen, L. Hutin, R. Lee, T.-J. K. Liu, and V. Stojanovic, "Relays do not leak- CMOS Does" Proceedings of the 50th Annual Design Automation Conference on - DAC 13, 2013.

[7] S. C. Masmanidis, R. B. Karabalin, I. De Vlaminck, G. Borghs, M. R. Freeman, and M. L. Roukes, "Multifunctional nanomechanical systems via tunably coupled piezoelectric actuation.", Science, vol. 317, pp. 780-783, 2007.

[8] S. Ilyas S, N. Jaber, and M. I. Younis, "MEMS logic using mixedfrequency excitation," J. MEMS, 2017.

[9] M. A. A. Hafiz, L. Kosuru, and M. I. Younis, "Microelectromechanical reprogrammable logic device," Nat. Commun., vol. 7, p. 11137, 2016.

[10] M. A. A. Hafiz, L. Kosuru, M. I. Younis, and H. Fariborzi, "Microelectromechanical resonator based digital logic elements," in ESSDERC/ESSCIRC 2016, Lausanne, Switzerland.

[11] M. A. A. Hafiz, S. Ilyas, S. Ahmed, M. I. Younis, and H. Fariborzi, “A Microbeam Resonator With Partial Electrodes for Logic and Memory Elements," IEEE Journal on Exploratory Solid-State Computational Devices and Circuits, vol. 3, pp. 83-92, 2017.

[12] J. S. Wenzler, T. Dunn, T. Toffoli, and P. Mohanty, “A nanomechanical fredkin gate,” Nano Lett., vol. 14, pp. 89-93, 2014.

[13] M. Shoba and R. Nakkeeran, "GDI based full adders for energy efficient arithmetic applications," Engineering Science and Technology, an International Journal, vol. 19, no. 1, pp. 485-496, 2016.

[14] M. I. Younis, MEMS Linear and Nonlinear Statics and Dynamics. New York, NY, USA: Springer, 2011.

[15] "COMSOL Multiphysics Reference Manual, version 5.2", COMSOL, Inc, www.comsol.com.

[16] J. M. Rabaey, Digital Integrated Circuits: A Design Perspective, Englewood Cliffs, NJ:Prentice-Hall, 1995.

[17] H. F. Dadgour, M. M. Hussain, A. Cassell, N. Singh, and K. Banerjee, "Impact of scaling on the performance and reliability degradation of metal-contacts in NEMS devices," 2011 International Reliability Physics Symposium, 2011. 